\title{
Predictive thermal control applied to HabEx
}

\author{
Thomas E. Brooks*a \\ ${ }^{a}$ NASA's Marshall Space Flight Center, AL 35812;
}

\begin{abstract}
Exoplanet science can be accomplished with a telescope that has an internal coronagraph or with an external starshade. An internal coronagraph architecture requires extreme wavefront stability (10 pm change/10 minutes for $10^{-10}$ contrast), so every source of wavefront error (WFE) must be controlled. Analysis has been done to estimate the thermal stability required to meet the wavefront stability requirement. This paper illustrates the potential of a new thermal control method called predictive thermal control (PTC) to achieve the required thermal stability. A simple development test using PTC indicates that PTC may meet the thermal stability requirements. Further testing of the PTC method in flight-like environments will be conducted in the X-ray and Cryogenic Facility (XRCF) at Marshall Space Flight Center (MSFC).
\end{abstract}

Keywords: Thermal stability, thermal analysis, thermal control system, wavefront stability, HabEx, coronagraph, mirror, thermal soak, thermal expansion, ULE, surface figure error, finite element analysis, CTE

\section{INTRODUCTION}

Exoplanet science (with an internal coronagraph) requires extreme wavefront stability (10 pm change/10 minutes), so every source of wavefront error (WFE) must be tightly controlled and extremely stable. Passive stability is certainly preferable for simplicity, reliability, and cost, but the requirements are so stringent that passive insulation may not allow the telescope to efficiently perform science. This paper decomposes the $10 \mathrm{pm} / 10$ minute stability requirement into thermal requirements. A test uses PTC to control a mirror's temperature, and the results indicate that PTC may meet the thermal stability requirements. As a point of departure, the wavefront of the Hubble Space Telescope (HST) fluctuates by about $30 \mathrm{~nm}$ over its 90 minute orbit, or 3,333pm per 10 minutes. ${ }^{[1]}$ Note that HabEx has baselined Lagrange point 2 (L2) as HabEx's orbit, and L2 has greater thermal stable than HST's orbit.

\section{THERMAL STABILITY REQUIREMENTS}

Thermal disturbances cause a telescope's optics to bend and translate relative to one another. Both of these effects cause the system's wavefront to change. A condensed structural, optical, and thermal (STOP) analysis has been performed to estimate how rapidly the mirror bends due to thermal gradients. The telescope in Figure 1 was analyzed at L2. The model assumes that a control system on the telescope's shroud controls the shroud to a set point by adjusting heater outputs periodically. This results in the shroud's temperature cycling by some amplitude (termed the "temperature fluctuation") and with some period as shown in Figure 2. As the shroud's temperature fluctuates, the mirror's temperature fluctuates which causes the mirror's surface to change. Interesting linear relationships are found between the telescope's design parameters and its

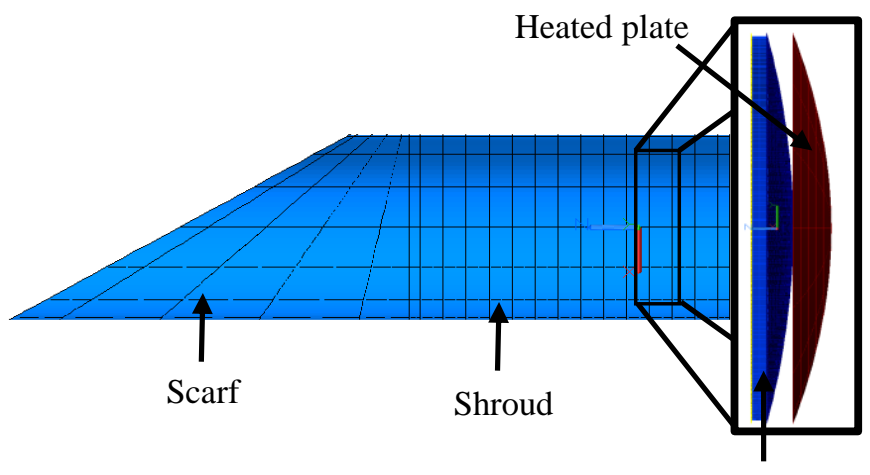

Mirror

Figure 1: Telescope Model. An enlarged view of the primary mirror and heated plate are shown on the right.

thermal stability ${ }^{[2]}$ Most importantly, the required quality of the shroud's thermal control system has been determined for this telescope and is graphically represented in Figure 3.

*thomas.brooks@nasa.gov;

(256)797-3147;

nasa.gov 


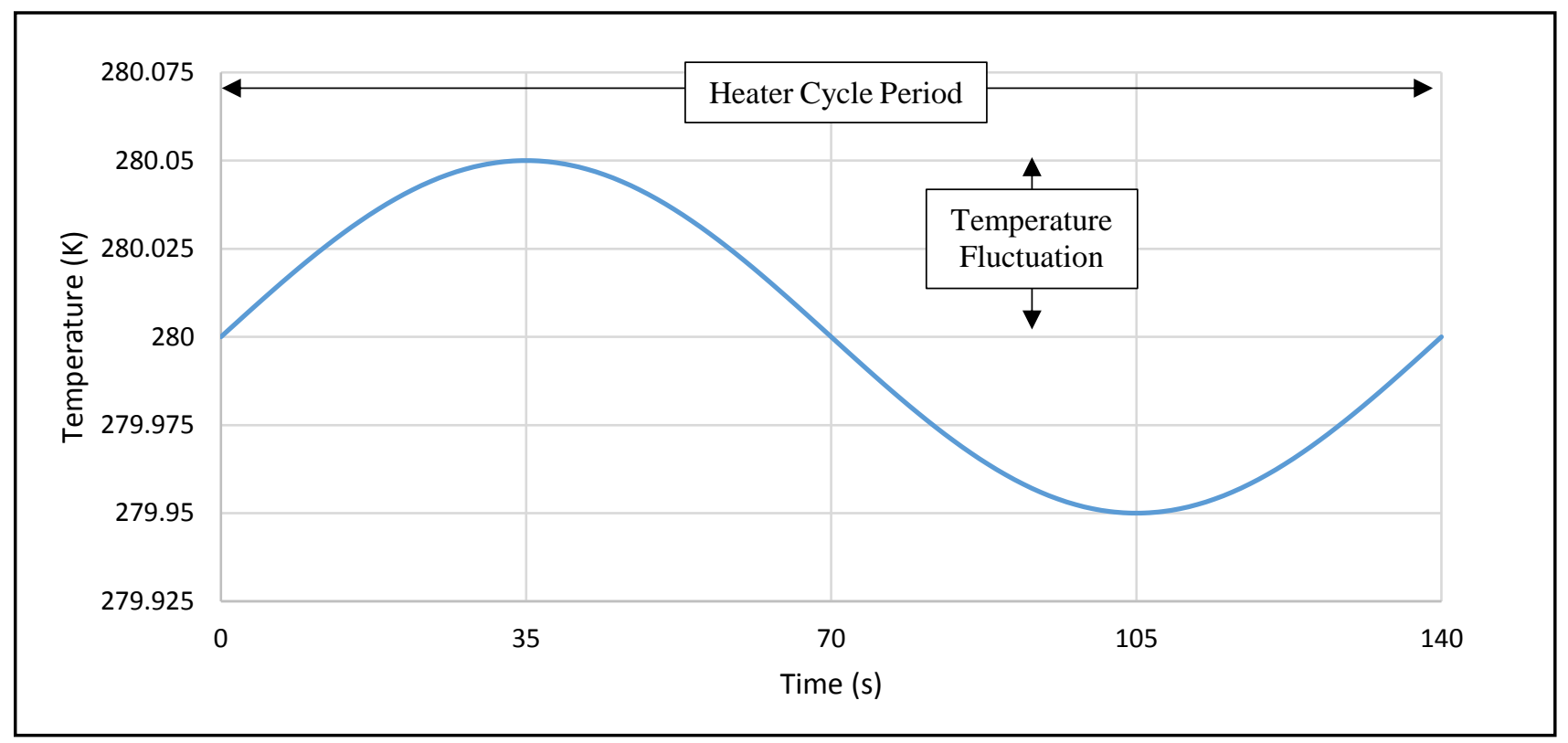

Figure 2: Temperature of the shroud and scarf. In this example, the temperature fluctuation is $50 \mathrm{mK}$ and the period is $140 \mathrm{~s}$.

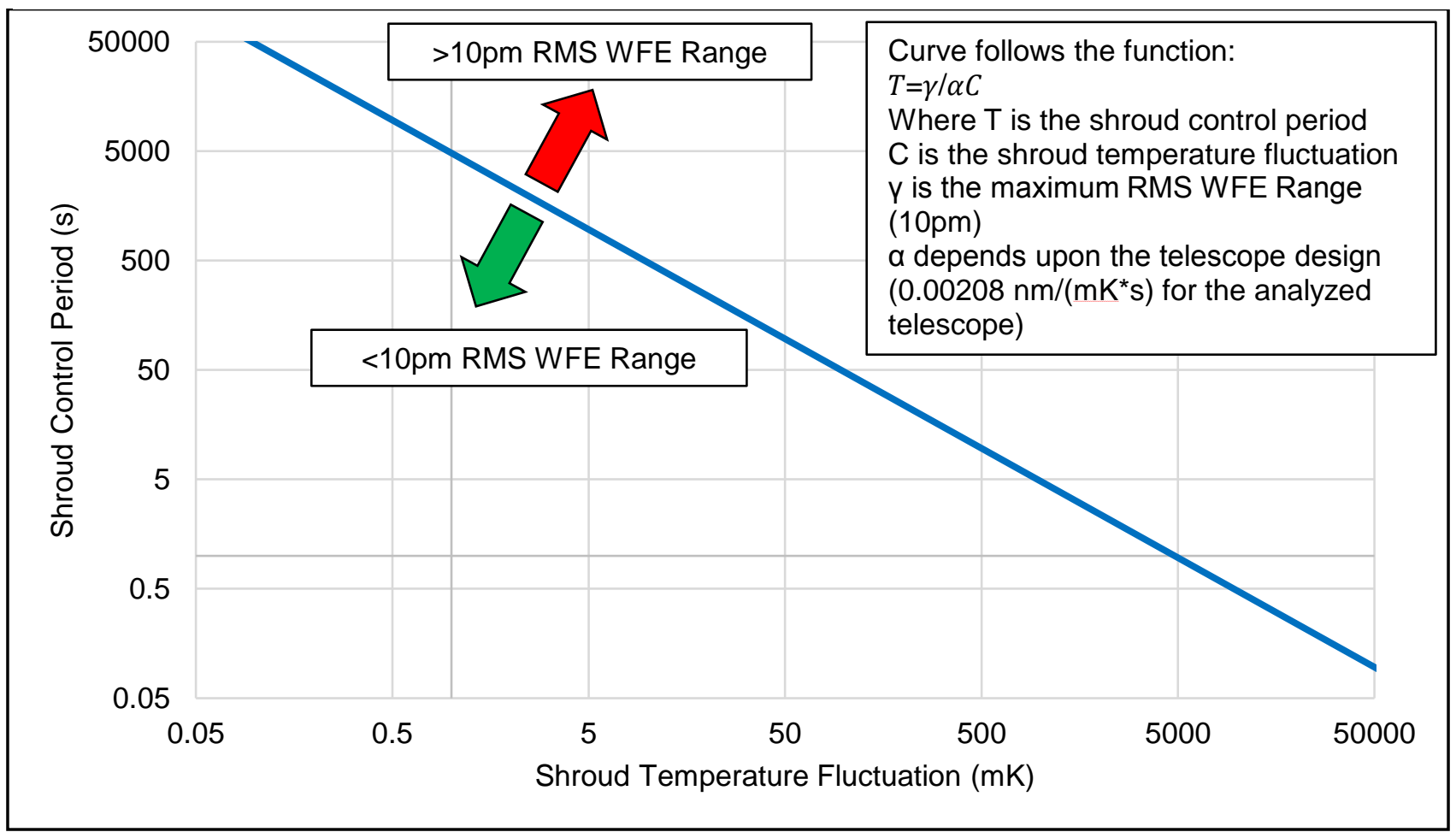

Figure 3: Shroud thermal requirements. The rate of change in the telescope's RMS WFE range is proportional to the telescope shroud's control period and temperature fluctuation. The RMS WFE range is also a function of the telescope's passive thermal design. As long as the shroud's thermal control system has a period and temperature fluctuation that is below the blue curve, the thermal control system will provide a sufficiently stable wavefront. 
The equation presented in Figure 3 deserves further explanation because of the impact which it may have on the telescope's thermal design. The equation is expressed again in a rearranged format in Equation 1.

$$
\gamma=T \alpha C
$$

Equation 1

This equation compresses the effects of the entire passive thermal design into one value that is represented with $\gamma \cdot \gamma$ certainly has many dependencies which can be used to improve the telescope's passive thermal design. Several of these dependencies have been previously documented, and are expressed in Equation 2. ${ }^{[2]}$

$$
\gamma=\frac{C T E}{\rho c_{p}} \gamma_{\text {remaining }}
$$

Where $\rho$ is the mirror's density, $c_{p}$ is the mirror's specific heat, CTE is the mirror's coefficient of thermal expansion, and $\gamma_{\text {remaining }}$ includes other terms that are not otherwise captured (and won't have a simple, linear relationship with RMS WFE range) like emissivities, linear conductors, telescope structural masses and specific heats, etc. From this equation, it is clear that a primary mirror has a lower RMS WFE range (greater optothermal stability) when it has a greater heat capacitance and a lower CTE.

\section{PREDICTIVE THERMAL CONTROL METHOD}

The predictive thermal control system has at its core a new algorithm which employs the model predictive control method. Instead of being reactive to temperature measurements, the predictive thermal control system predicts what temperatures will be at the next time step (one heater cycle period in the future) and adjusts the heater output, so that the system's temperature distribution is the desired temperature distribution. This proactive thermal control approach is expected to be better than the reactive thermal control approach commonly used today by proportional, integral, and derivative (PID) control logic. The PTC also takes into account how one control volume's temperature affects all other control volume's temperature, and the fact that heat transfer in a vacuum has a non-negligible non-linear $\left(\mathrm{T}^{4}\right)$ radiative component. A conventional proportional control system calculates heat output by following Equation 3 where $Q_{i}$ is the heat output at location i, $K_{p}$ is the proportional coefficient, $T_{d, i}$ is the desired temperature at location $\mathrm{i}$, and $T_{m, i}$ is the measured temperature at location $\mathrm{i}$.

$$
Q_{i}=K_{p} *\left(T_{d, i}-T_{m, i}\right)
$$

Equation 3

The PTC algorithm uses Equation 4 where $C_{i}$ is the heat capacitance of volume $\mathrm{i}, \mathrm{n}$ represents the time step, $\mathrm{T}$ is temperature, $\mathrm{t}$ is time, $\mathrm{Q}$ is an internal heat load (heaters, electronics, chemical reactions, nuclear radiation, etc), $G_{j i}$ is a linear conductor (between volume $\mathrm{i}$ and $\mathrm{j}$ ) and $\widehat{G_{\jmath l}}$ is a radiative conductor (between volume $\mathrm{i}$ and $\mathrm{j}$ ).

$$
\begin{array}{rlrl}
\frac{2 C_{i}}{\Delta t}\left(T_{i}^{n+1}-T_{i}^{n}\right) & =2 Q_{i} & \\
& +\sum_{j=1}^{N}\left[G_{j i}\left(T_{j}^{n}-T_{i}^{n}\right)+\widehat{G_{j l}}\left\{\left(T_{j}^{n}\right)^{4}-\left(T_{i}^{n}\right)^{4}\right\}\right] & & \text { Equation } 4 \\
& +\sum_{j=1}^{N}\left[G_{j i}\left(T_{j}^{n+1}-T_{i}^{n+1}\right)+\widehat{G_{j l}}\left\{\left(T_{j}^{n+1}\right)^{4}-\left(T_{i}^{n+1}\right)^{4}\right\}\right] &
\end{array}
$$

This equation is used in commercial thermal solvers like SINDA/FLUINT. ${ }^{[3]}$ The control system measures the temperatures, so they are known. $\Delta \mathrm{t}$ is the control period which is set by the control system operator. The equation's use requires the determination of the conductors and heat capacitances, and it can be solved iteratively or by linearizing the radiative conductors at every time step. The Qs are solved for and the required voltage is calculated based upon the heaters' resistance. Finally, the control system commands the power supplies to supply the voltage that will result in the Qs. 


\section{PREDICTIVE THERMAL CONTROL TEST}

The deep core mirror (made as a technology demonstrator) was used as the test article. Heaters and thermocouples (calibrated in house) were taped to the deep core mirror with Kapton tape as shown in Figure 4. There are nine thermocouples on the back of the mirror and nine thermocouples on the mirror's sides (three every $120^{\circ}$ - one at the front, one at the middle, and one at the back). Heaters are placed on the side at the same location as the middle thermocouple. One heater patch is circled in red in Figure 4.

The mirror's temperature distribution was controlled using the three heaters that are on the mirror's sides. The PTC algorithm described in section 3 was employed with an 18 channel data acquisition (DAQ) system and three separate power supplies. The DAQ and power supplies were connected to a laptop and all of the temperature monitoring and power control was handled with Python code written in house.

The mirror sits on a v-block and it is initially at ambient temperature and always at ambient pressure. The v-mount has low conductivity foam pads (light brown in Figure 4) and those pads (along with interface resistances) limit conductive heat transfer from the mirror to the v-block. The primary heat transfer method between the mirror and its environment is natural convection where hot spots on the mirror heat up the surrounding air and cause the air to expand (lowering its density) and drift upward to carry the heat away. Initially, the mirror is essentially at a steady

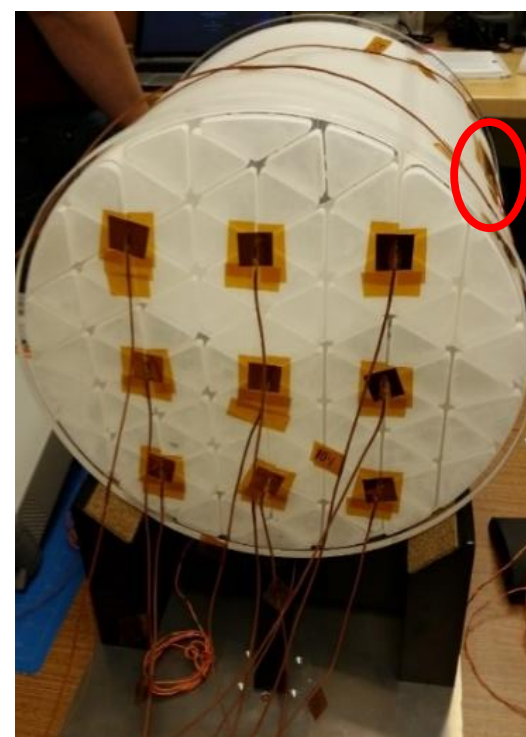

Figure 4: Deep core mirror with thermocouples and heaters applied with Kapton tape.

state for its surrounding environment, but as the test begins, the power supplies, DAQ, and laptop are turned on which causes the room temperature to increase by a measureable amount. These effects were not put into the model which drives the PTC algorithm, yet the system worked well with this unknown. Several tests were performed to develop the PTC algorithm, and one test dataset is shown in Figure 5. The algorithm is simply told to make the temperature at those control zones $320 \mathrm{~K}$, and this is what results.

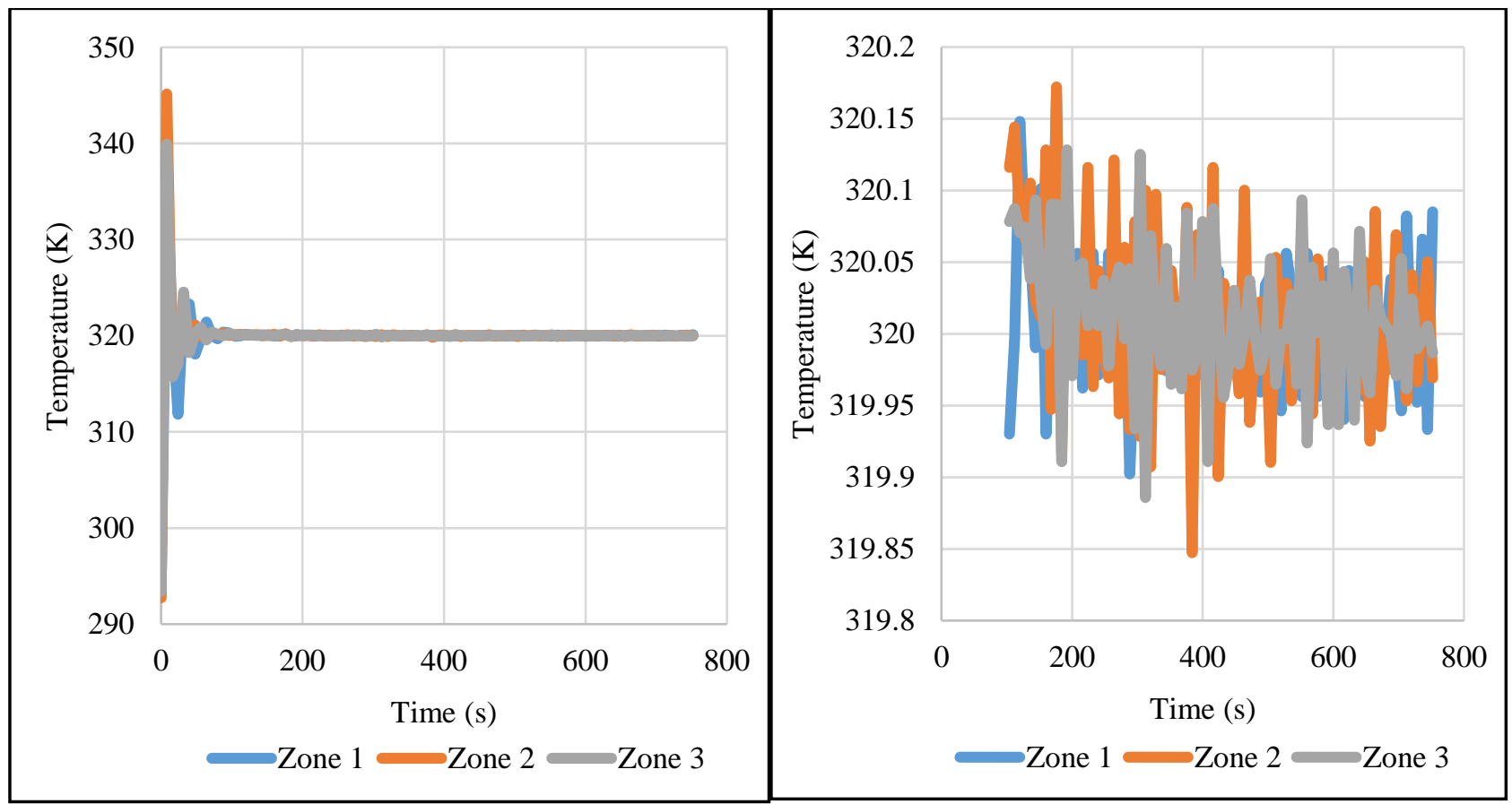

Figure 5: Left: Temperature data taken at the three control zones when the control period is 8 seconds. Right: Temperature data when omitting the initial transient 
The control system ran at control periods ranging from 2-10 seconds. It was found that the standard deviation of the control zone temperature changed as the control period changed. The relationship between the control period and the standard deviation of the temperatures is shown in Figure 6. The yellow dashed line shows the RMS WFE range that analysis indicates the telescope will experience with this control system.

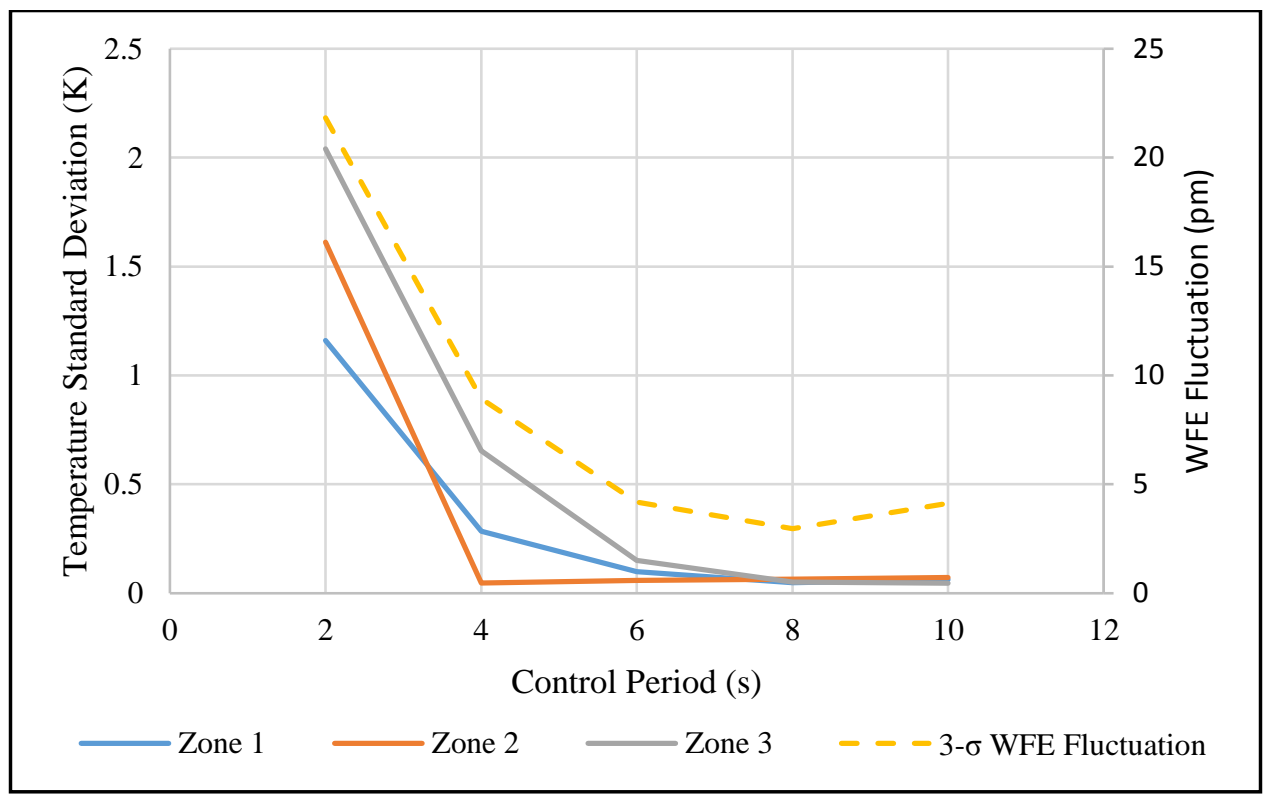

Figure 6: The standard deviation of the temperatures on the control zones and an estimate of the resulting RMS WFE range.

Shorter control periods have greater temperature standard deviations. A control system works best when the control period is short and the standard deviation of the temperature is small, so it is beneficial to have a shorter control period without hurting the temperature standard deviation. The problem that causes the temperature standard deviation to increase at short control periods is shown graphically in Figure 7.

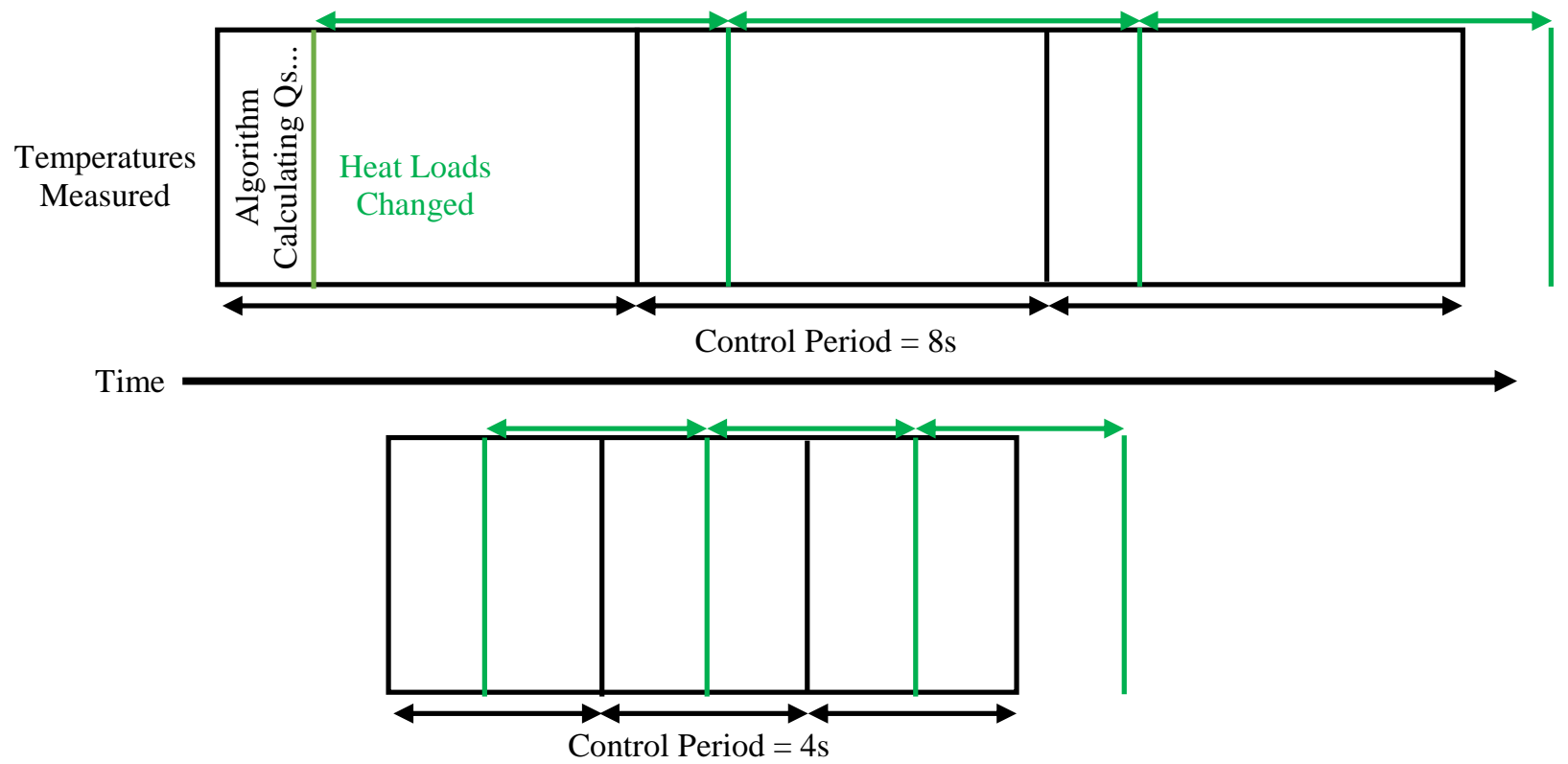

Figure 7: A graphical representation of the lag between the temperature measurement cycle (black) and the heat load adjustment cycle (green). The top image shows three periods of heater cycles with a control period of $8 \mathrm{~s}$ and the bottom image shows three heater cycle periods with a control period of $4 \mathrm{~s}$. 
The green vertical lines represent times at which the heat loads are commanded to change and the black vertical lines represent times at which the temperature is measured. When the control period becomes too short, the time that it takes to calculate new heat loads becomes a sizeable portion of the control period. This lag between the measurement time and the heat load adjustment time increases the temperatures' standard deviation. The best way to reduce this error is to reduce the PTC's run time. The currently written PTC code solves the heat equation in parallel, but is otherwise not optimized for efficiency, and it is written an interpreted language (Python). Interpreted languages typically have slower execution times than compiled languages. The code could certainly be written to run faster. Another option to reduce run time is to reduce the number of control volumes in the thermal model that is imbedded within the PTC.

Even with these known and improvable deficiencies in the implementation of the PTC algorithm, the algorithm provides enough thermal stability for the tested case. A comparison between the test results and the requirements is shown in Figure 8 .

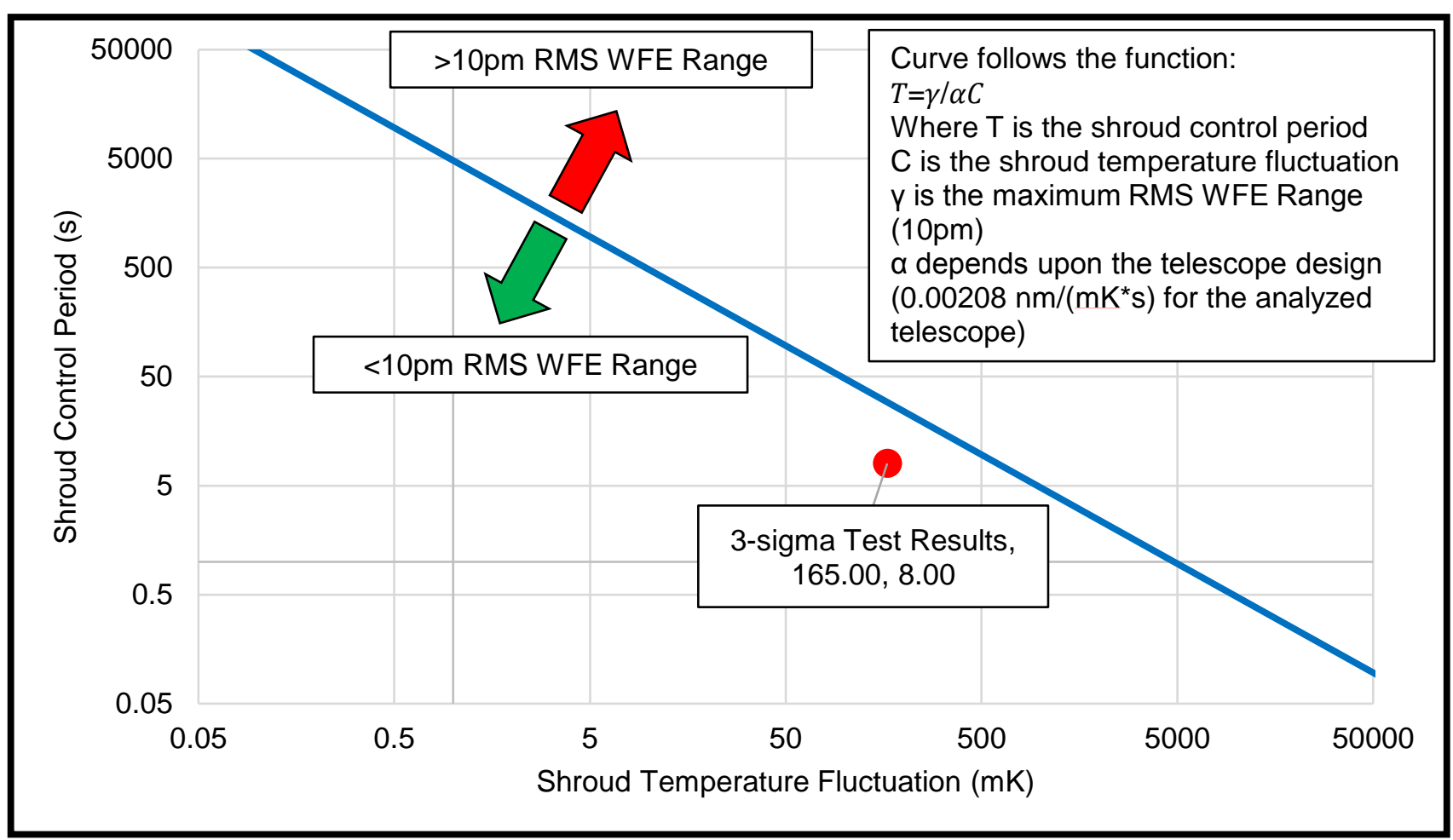

Figure 8: Comparison between test results and thermal stability requirements.

\section{CONCLUSION}

An internal coronagraph can achieve $10^{-10}$ contrast as long as a telescope provides a wavefront that does not change by more than $10 \mathrm{pm} / 10$ minutes. This requirement has been turned into a thermal stability requirement that is illustrated graphically in Figure 3 and Figure 8. A new thermal control algorithm met the thermal stability requirements in recent development tests with low-cost, high TRL heaters and thermocouples. 


\section{REFERENCES}

[1] Cox, Lallo "Keeping the Hubble Space Telescope in focus", Proc. SPIE 8442, Space Telescopes and Instrumentation 2012: Optical, Infrared, and Millimeter Wave, 844237 (August 22, 2012): doi:10.1117/12.924900; http://dx.doi.org/10.1117/12.924900

[2] Brooks, et al "Advanced Mirror Technology Development (AMTD) thermal trade studies", Proc. SPIE 9577, Optical Modeling and Performance Predictions VII, 957703 (September 23, 2015); doi:10.1117/12.2188371; http://dx.doi.org/10.1117/12.2188371

[3] C\&R Technologies, "User's Manual June 2015 SINDA/FLUINT,” C\&R Technologies website, September 2015, https://www.crtech.com/sites/default/files/files/Guides-Manuals/Protected/sf58main.pdf (September 2015). 